\title{
因HAD
}

ISSN-L: 2530-5115

DOI: http://doi.org/10.22585/hospdomic.v1i3.23

\section{YouTube como fuente de información sanitaria y divulgación del conocimiento sobre Cuidados Paliativos en el domicilio}

\section{YouTube as a source of health information and knowledge about Palliative Care at home}

\author{
Vicent Oller-Arlandis', Vanessa Esperanza Oller-Arlandis² \\ 1. Coordinador Médico UHD Hospital de Manises, Valencia, España \\ 2. Departamento de Salud Pública, Historia de la Ciencia y Ginecología, Universidad Miguel Hernández, \\ Alicante, España.
}

Correspondencia/Correspondence

Vanessa E. Oller-Arlandis

Departamento de Salud Pública, Historia de la

Ciencia y Ginecología.

Universidad Miguel Hernández

Ctra. Alacant-València km.8.7, 03016 , Sant

Joan d'Alacant, Alicante, España.

vaneollerarlandis@gmail.com

Recibido/Received

26.06.2017

Aceptado/Accepted

30.06.2017
Conflicto de Intereses/Competing interest

El autor y la autora declaran la inexistencia de cualquier conflicto de interés.

Financiación/Fundings

No se ha recibido financiación específica para publicar este trabajo.

\section{CÓMO CITAR ESTE TRABAJO | HOW TO CITE THIS PAPER}

Oller-Arlandis V, Oller-Arlandis VE. YouTube como fuente de información sanitaria y divulgación del conocimiento sobre Cuidados Paliativos en el domicilio. Hosp Domic. 2017;1 (3): 153-66. 


\section{RESUMEN}

Objetivo: Describir las características del material multimedia sobre cuidados paliativos en el domicilio disponible en YouTube, y evaluar la calidad de la información contenida en este material.

Método: Estudio descriptivo de la información recuperada en YouTube. Los vídeos se obtuvieron mediante búsqueda simple utilizando como término «cuidados paliativos en el domicilio», determinándose su número mediante muestreo aleatorio simple sin reposición. Las características de los vídeos se estudiaron mediante los indicadores: producción/año, actualidad, duración, número de reproducciones o visionados, e interacción del usuario.

Resultados: De los 388 vídeos seleccionados, se pudieron analizar un total de 204 que presentaron un crecimiento lineal de $R 2=0,04 ; p$ $=0,005$. El número medio de visionados fue de 8.497,9 9 4.372,09 con mediana de 298 consultas. La obsolescencia presentó una mediana de 3 años. La interacción de usuario se presentó de media con 31,97 \pm 13,91 indicaciones favorables, $2,98 \pm 1,36$ comentarios y $10.271,00 \pm$ $1.768,15$ suscripciones.

Conclusiones: Las características del material multimedia fueron: gran producción y divulgación (consulta), rápida obsolescencia y una escasa participación de usuario. La mayoría de los videos analizados pueden considerarse adecuados con el tema a estudio.

Palabras clave: Cuidados paliativos; Cuidados Paliativos al final de la vida; Servicios de Atención a Domicilio Provisto por Hospital; Internet; Red social; Medios de Comunicación Sociales; Diseminación de información; Control de calidad; YouTube.

\section{ABSTRACT}

Objective: To describe the characteristics of multimedia material on palliative care at home available on YouTube, and to assess the quality of the information contained in this material.

Methods: This was a descriptive study of the information retrieved from YouTube. Videos were identified by means of a simple search using the term "cuidados paliativos en el domicilio" and simple random sampling without replacement was employed to select the study sample. Video characteristics were described by the following indicators: production/year, currency, length, number of views and user interaction.

Results: Among the three hundred and eightyeight videos selected, we could analyze a total of 204 that presented a linear growth of R2 = $0.04 ; P=0.005$. The mean number of views was $8497.9 \pm 4372.09$ with a median of 298 visits. The obsolescence presented a median of 3 years. The user interaction was presented on average with $31.97 \pm 13.91$ favorable indications, $2.98 \pm 1.36$ comments and $10271.00 \pm$ 1768.15 subscriptions.

Conclusions: The multimedia material studied presented the following characteristics: largescale production and dissemination (views), rapid obsolescence and scant user participation. Most of the analyzed videos can be considered adequate to the subject of the study.

Keywords: Palliative Care; Hospice care; Home care services, hospital-based; Internet; Social networking; Social communication; Information Dissemination; quality control; YouTube. 


\section{INTRODUCCIÓN}

Basadas en Internet, y en las aplicaciones de la Web 2.0, las redes sociales son comunidades online de individuos u organizaciones que comparten información, datos, gustos, aficiones y/o intereses en común muy diversos. Estas son, por su propia naturaleza interactiva, el medio de comunicación más popular y creciente entre los millones de usuarios de Internet. Es por ello que, hoy día, las redes sociales como YouTube, Facebook, Twitter, etc., se han convertido en importantes y valiosas herramientas para la comunicación social, la transmisión e intercambio ágil de información o, incluso, para la educación.

Además, estas redes virtuales, representan una gran oportunidad para la investigación de los contenidos presentes en la Red sobre distintas áreas de la ciencia, la medicina y la salud pública, entre otras materias (1).

Así, desde que naciera en el año 2005, la plataforma social para compartir vídeos, YouTube, es uno de los referentes de la Web $2.0(1,2)$, siendo la segunda web con mayor tráfico de visitas en España y la segunda en el mundo en mayo de $2017(3,4)$.

El fenómeno YouTube ha participado en la expansión exponencial de contenidos audiovisuales, democratizando el acceso al material multimedia y facilitando que los propios usuarios se conviertan en verdaderos generadores de contenidos (4).

Los más de mil millones de usuarios de YouTube (5) pueden ser simples espectadores en línea del material multimedia disponible en la plataforma y/o creadores (también denominados "Youtubers") de dichos contenidos. Así, este sitio Web se ha convertido en un medio de difusión y promoción tan popular que políticos, organizaciones de todos los ámbitos (gubernamentales y no gubernamentales) e incluso celebrities (artistas, personajes importantes, famosos...) han dejado claro como las tecnologías de la información y de la comunicación (TIC) han modificado la dimensión social en muchos aspectos de la vida. Pero, el problema, hoy día, no es encontrar información, sino saber seleccionar la más relevante entre el exceso de mensajes incompletos, inconexos e inexactos (6).

Por eso, se ha creído necesario conocer como se está transmitiendo a través de las herramientas 2.0 la información sobre Cuidados Paliativos en el domicilio.

En consecuencia, el principal objetivo de este trabajo es evaluar el cumplimiento de las variables de calidad del material multimedia sobre Cuidados Paliativos en el domicilio contenido en YouTube y como objetivo secundario describir las características de este material y analizar la interacción que los usuarios realizan con los mismos.

\section{MATERIAL Y MÉTODO}

Estudio descriptivo transversal de la información recuperada sobre Cuidados Paliativos en el domicilio a partir de los vídeos contenidos en la edición española de YouTube.

Se consideró el cálculo de predicción del buscador de YouTube. Este buscador nunca ofrece un resultado mayor a 500 vídeos pero "estima" el número que deben existir según el tiempo empleado en reconocer los 500 primeros.

Para la obtención de este material multimedia se realizó una búsqueda simple utilizando como término "cuidados paliativos en el domicilio", determinándose el número a estudio mediante muestreo aleatorio simple sin reposición, efectuando la estimación de parámetros poblacionales (valor 
esperado aproximado a 0,5, precisión del intervalo 0,05 y nivel de confianza $=0,95$ ) en una población infinita.

Se seleccionaron 388 vídeos; 386 mediante muestreo aleatorio simple sin reemplazo, más las 2 referencias no incluidas en esta muestra pertenecientes a los 20 primeros vídeos (primera pantalla) identificados para el término de búsqueda que, también, se incluyeron en el estudio. Para evitar modificaciones en los resultados y mantener el enlace a los vídeos seleccionados, se almacenó la dirección electrónica de cada uno de ellos en una hoja de cálculo Excel 2007@ que facilitó el hipervínculo a la URL y su acceso permanente, los cálculos matemáticos y la construcción de tablas y figuras. Los cálculos estadísticos, se realizarán empleando el programa SPSS $®$ versión 15.0 para Windows. La fecha final del visionado de los vídeos fue mayo de 2017.

Para caracterizar el material multimedia se utilizaron las siguientes variables descriptivas:

- Accesibilidad: validez de acceso al vídeo a estudio en el momento de enlazar.

- Fecha de subida: día en el que el vídeo se incluyó en YouTube (año de publicación).

- Duración: tiempo que dura o que transcurre entre el comienzo y el fin del proceso de visionado.

- Reproducciones: número de visionados del vídeo.

- Indicaciones favorables: número de veces en las que el vídeo fue evaluado positivamente por la persona que lo visionó ['me gusta'].

- Indicaciones desfavorables: número de veces en las que el vídeo fue evaluado negativamente por la persona que lo visionó ['no me gusta'].

- Comentarios: número de comentarios que figuran en la página del vídeo.

- Comentarios positivos: número de comentarios favorables que figuran en la página del vídeo.

- Suscripciones: número de personas que se han abonado al vídeo.

- Actualidad/obsolescencia: tiempo (años) trascurrido entre la fecha de subida y la fecha de estudio.

- Autoría: identificación de la persona o institución (filiación) responsable de la subida del vídeo.

- Profesional: tipo de profesional dedicado a la asistencia en Cuidados Paliativos (CP) que aparece en el vídeo a estudio.

- Paciente: tipo de paciente (rango de edad) que aparece o sobre el que versa el vídeo a estudio.

- Enfermedad: tipo de enfermedad crónica de base que se describe en el vídeo.

- Tema del vídeo: contenido informativo principal del vídeo subido a YouTube en relación con el tema a estudio.

- Coherencia del título: existencia, relación y validez del nombre del vídeo en relación a la temática de contenidos.

- Adecuación del contenido: corrección de la información del material multimedia en relación con los CP.

- Publicidad: presencia de mensajes publicitarios durante el proceso de visionado del vídeo.

En el análisis de los datos, las variables cualitativas se describieron por su frecuencia y porcentaje, las cuantitativas mediante su media y desviación estándar, representando las más relevantes mediante la utilización de tablas y gráficos.

Se utilizó la media y la mediana, como medidas de tendencia central, calculándose también, el máximo y el mínimo. Para determinar el tipo de crecimiento de la producción científica se utilizó la 
regresión con ajuste de la curva. El nivel de significación utilizado en todos los contrastes de hipótesis fue $a \leq 0,05$ con un Intervalo de confianza del 95\%.

\section{RESULTADOS}

A la hora de realizar el estudio se observó que, de los 388 vídeos seleccionados, 4 (1,03\%; IC95\%: $0,03-2,04)$ ya no eran accesibles. Se detectó la presencia de 6 vídeos duplicados (1,55\%; IC95\%: $0,32-2,77)$ y 174 vídeos (44,85\%; IC95\%: 39,90 - 49,79) no estaba relacionados con el tema a estudio (cuidados paliativos en el domicilio). Así, quedaron disponibles para su estudio y análisis 204 vídeos (52,58\%; IC95\%: 47,61 - 57,55). Cabe señalar, de todos modos, que 10 de estos vídeos (4,90\%; IC95\%: 1,94 - 7,86) no trataron específicamente sobre el tema objeto del estudio, más bien serían cuidados enfermeros en el domicilio. Considerándose adecuados un total de 194 videos (95,10\%; IC95\%: 92,14-98,06).

En 151 (74,02\%; IC95\%: 68,00 - 80,04) de los vídeos a estudio, se observó coherencia entre el título mismo y el tema a estudio.

Se comprobó una evolución ascendente en el número de vídeos subidos a YouTube, acreditando que el modelo con un mayor ajuste fue el lineal a lo largo del tiempo (coeficiente de determinación R2 = 0,04; $\mathrm{p}=0,005$ ); ver figura 1 .

Las principales características del material multimedia estudiado pueden consultarse en tabla 1.

Figura 1. Evolución temporal de la producción multimedia en YouTube sobre Cuidados Paliativos en el Domicilio

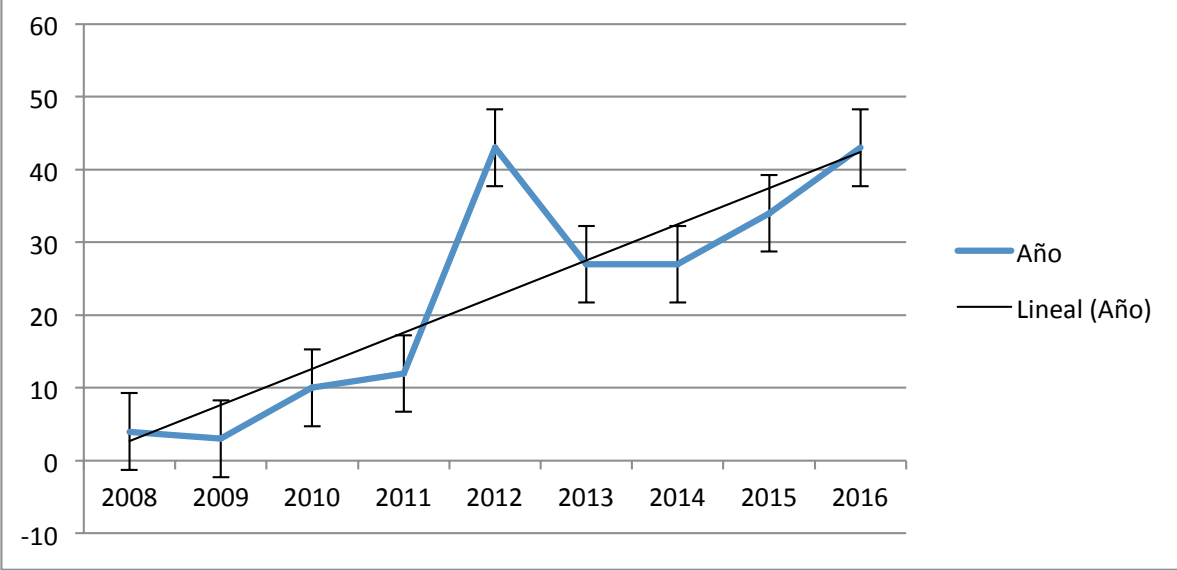




\begin{tabular}{|c|c|c|c|c|}
\hline Indicador & Máximo & Mínimo & Media \pm DS & Mediana \\
\hline Actualidad/obsolescencia (años) & 9 & 1 & $3,52 \pm 0,14$ & 3 \\
\hline Duración (hh:mm:ss) & $5: 28: 05$ & 00:00:05 & $00: 14: 52 \pm 00: 02: 24$ & 00:03:42 \\
\hline Número de reproducciones & 772.925 & 6 & $8.497,91 \pm 4.372,09$ & 298 \\
\hline Indicaciones favorables & 1.998 & 0 & $31,97 \pm 13,91$ & 2 \\
\hline Indicaciones desfavorables & 202 & 0 & $1,99 \pm 1,07$ & 0 \\
\hline Comentarios & 223 & 0 & $2,88 \pm 1,36$ & 0 \\
\hline Comentarios positivos & 125 & 0 & $2,19 \pm 0,97$ & 0 \\
\hline Suscripciones & 174.225 & 0 & $10.271,00 \pm 1.768,15$ & 417 \\
\hline
\end{tabular}

Para conocer los datos de participación de los usuarios se calculó el cociente de las indicaciones favorables o desfavorables por número de visualizaciones. Igualmente, se realizó la misma operación para el número de comentarios, de comentarios favorables y de suscripciones a los vídeos, ver tabla 2.

\begin{tabular}{|c|c|c|}
\hline & Cociente $^{*}$ & Cociente por mil \\
\hline Indicaciones favorables & 0,00376 & 3,76 \\
\hline Indicaciones desfavorables & 0,00023 & 0,23 \\
\hline Comentarios & 0,00034 & 0,34 \\
\hline Comentarios favorables & 0,00026 & 0,26 \\
\hline $\begin{array}{l}\text { * Indicador estudiado dividio } \\
\text { Total reproducciones igual a }\end{array}$ & & \\
\hline
\end{tabular}

Los datos sobre la autoría pueden consultarse en la tabla 3. 


\begin{tabular}{|l|r|r|}
\hline \multirow{2}{*}{ Tabla 3: Autoría de los vídeos sobre Cuidados Paliativos en el domicilio estudiados. } \\
\hline & \multicolumn{2}{|c|}{ Autoría } \\
\cline { 2 - 3 } & $\mathbf{f}_{\mathbf{0}}$ & \multicolumn{2}{|c|}{ \% (IC95\%) } \\
\hline Profesional socio-sanitario & 31 & $15,20(10,27-20,12)$ \\
\hline Medios de comunicación & 31 & $15,20(10,27-20,12)$ \\
\hline Persona privada & 31 & $15,20(10,27-20,12)$ \\
\hline Hospital/Institución sanitaria & 23 & $11,27(6,93-15,61)$ \\
\hline Asociación de enfermos & 15 & $7,35(3,77-10,93)$ \\
\hline Fundación & 15 & $7,35(3,77-10,93)$ \\
\hline Colegio profesional & 12 & $5,88(2,65-9,11)$ \\
\hline Empresa privada de servicios & 11 & $5,39(2,29-8,49)$ \\
\hline Administración sanitaria local & 9 & $4,41(1,59-7,23)$ \\
\hline Desconocido & 8 & $3,92(1,26-6,59)$ \\
\hline Universidad & 7 & $3,43(0,93-5,93)$ \\
\hline Administración gubernamental & 4 & $1,96(0,06-3,86)$ \\
\hline Sociedad médica & 3 & $1,47(0,00-3,12)$ \\
\hline Centro de formación & 3 & $1,47(0,00-3,12)$ \\
\hline Organización no gubernamental & 1 & $0,49(0,00-1,45)$ \\
\hline
\end{tabular}

Por otro lado, en cuanto al tema principal que se trata en cada uno de los vídeos estudiados, los datos obtenidos quedan plasmados en la tabla 4 .

\begin{tabular}{|l|r|r|}
\hline \multirow{2}{*}{ Tabla 4: Tema principal de los vídeos sobre Cuidados Paliativos estudiados tras el visionado. } \\
\hline CP en el domicilio & $\mathbf{f}_{0}$ & \multicolumn{2}{|c|}{ Tema } \\
\cline { 2 - 3 } & 114 & (IC95\%) \\
\hline CP en el hospital/domicilio & 22 & $55,88(49,07-62,70)$ \\
\hline CP en el hospital & 16 & $10,78(6,53-15,04)$ \\
\hline Noticias sobre CP & 14 & $7,84(4,15-11,53)$ \\
\hline Conferencia/Jornada sobre CP & 9 & $6,86(3,39-10,33)$ \\
\hline UHD-CP & 7 & $4,41(1,59-7,23)$ \\
\hline Técnicas/procedimientos en CP & 7 & $3,43(0,93-5,93)$ \\
\hline Cursos/Máster sobre CP & 5 & $3,43(0,93-5,93)$ \\
\hline CP y muerte digna & 2 & $2,45(0,33-4,57)$ \\
\hline Otros & 7 & $0,98(0,00-2,33)$ \\
\hline No consta & 1 & $3,43(0,93-5,93)$ \\
\hline
\end{tabular}


Asimismo, se pueden consultar los datos sobre tipo de profesional de la salud, paciente y tipo de enfermedad tratada en la tablas 5,6 y 7 , respectivamente.

Señalar que se detectó la presencia de publicidad en 10 (4,90\% IC95\%: 1,94 - 7,86) de los vídeos sobre «Cuidados Paliativos en el domicilio» evaluados.

\begin{tabular}{|l|r|r|}
\hline \multirow{2}{*}{ Tabla 5: Profesional de Cuidados Paliativos en el domicilio implicado en los vídeos estudiados. } \\
\hline & \multicolumn{2}{|c|}{ Profesional } \\
\cline { 2 - 4 } & $\mathbf{f}_{0}$ & \multicolumn{2}{|c|}{ (IC95\%) } \\
\hline Equipo Interdisciplinar (ECP) & 81 & $39,71(32,99-46,42)$ \\
\hline Médico & 54 & $26,47(20,42-32,52)$ \\
\hline Enfermero/a & 29 & $14,22(9,42-19,01)$ \\
\hline Psicólogo/a & 6 & $2,94(0,62-5,26)$ \\
\hline Trabajador/a Social & 2 & $0,98(0,00-2,33)$ \\
\hline Voluntarios/as & 11 & $5,39(2,29-8,49)$ \\
\hline No consta & 21 & $10,29(6,12-14,46)$ \\
\hline
\end{tabular}

\begin{tabular}{|c|c|c|}
\hline & \multicolumn{2}{|c|}{ Paciente } \\
\hline & $f_{0}$ & $\%$ (IC95\%) \\
\hline Pediátrico (0 a 17) & 16 & $7,84(4,15-11,53)$ \\
\hline Adulto (18 a 64) & 14 & $6,86(3,39-10,33)$ \\
\hline Anciano/Adulto mayor (> 65) & 28 & $13,73(9,00-18,45)$ \\
\hline Todos los adultos (>18) & 52 & $25,49(19,51-31,47)$ \\
\hline TODAS las edades & 24 & $11,76(7,34-16,19)$ \\
\hline No consta & 70 & $34,31(27,80-40,83)$ \\
\hline
\end{tabular}




\begin{tabular}{|l|r|r|}
\hline \multirow{2}{*}{$\begin{array}{l}\text { Tabla 7: Enfermedad tratada/descrita en los vídeos sobre Cuidados Paliativos en el domicilio } \\
\text { estudiados. }\end{array}$} & \multicolumn{2}{|c|}{ Enfermedad } \\
\cline { 2 - 3 } & $\mathbf{f}_{\mathbf{0}}$ & (IC95\%) \\
\hline E.oncológica avanzada/ terminal & 72 & $35,29(28,74-41,85)$ \\
\hline Pulmonar (EPOC avanzado) & 2 & $0,98(0,00-2,33)$ \\
\hline Cardíaca (ICC sin opción) & 2 & $0,98(0,00-2,33)$ \\
\hline Neurológica (limitación severa) & 11 & $5,39(2,29-8,49)$ \\
\hline Demencias/Alzheimer & 4 & $1,96(0,06-3,86)$ \\
\hline SIDA (terminal) & 1 & $0,49(0,00-1,45)$ \\
\hline TODAS las descritas para CP & 36 & 76 \\
\hline No Consta & $17,65(12,42-22,88)$ \\
\hline
\end{tabular}

Reseñar que en los vídeos aparecieron mensajes en diversos formatos (noticias, entrevistas, conferencias, reportajes específicos, etc.) donde destacaba, tanto en los testimonios de pacientes y familiares como de los profesionales, el componente humano, la calidad de vida y el enfoque holístico. También se observaron contenidos con referencias a la gestión política, organizativa y de salud.

\section{DISCUSIÓN}

Si bien la recuperación de la información sobre salud en YouTube resulta a veces engañosa debido a la falta de etiquetas de búsqueda y taxonomías aceptadas para las listas de vídeos (7), la terminología en YouTube sobre cuidados paliativos en el domicilio tiene una importante presencia, a tenor de los resultados observados en el presente estudio.

Queda patente que los resultados de este trabajo prueban un pobre cumplimiento de los criterios relacionados con la calidad, ya que ningún vídeo satisfizo todos los ítems analizados. Quizá sean más indicativos los valores obtenidos medidos por la mediana, que en ninguno de los casos alcanzó la mitad de los criterios analizados.

El primer dato que afecta al estudio de la información sobre cuidados paliativos en el domicilio en YouTube es la pérdida del enlace. Así, «enlace roto» es un término informal que se utiliza cuando un sitio Web ya no puede ser consultado en Internet, generalmente porque su contenido ya no está almacenado en los servidores de manera permanente (muchas veces aparecerá como «error 404»). Cuando un usuario 'tropieza' con un enlace roto puede influir en el abandono de la búsqueda de esa información. De todos modos, el porcentaje de enlaces rotos observados en YouTube fue inferior al observado en los trabajos realizados en ciencias de la salud por Curbelo-Castro (8) de 
información sobre lactancia materna en YouTube o en los trabajos de Guardiola-Wanden-Berghe $(9,10)$ y Oller-Arlandis $(6)$ sobre la Web 2.0 , lo que indica que la muestra a estudio seleccionada tiene una adecuada representación.

La actualización de los contenidos en YouTube presentó un ritmo claramente ascendente. El modelo de ajuste lineal observado sigue los principios anunciados por Price (11) en el año 1956, ahora bien en un plazo temporal más corto (lo que antes suponía 15 años, ahora se cumple en menos de 3).

De los resultados obtenidos se desprende, también, que el alto número de reproducciones observado guarda relación con la clasificación Alexa (3), donde sitúa a YouTube como la segunda herramienta más utilizada de la Web 2.0 a nivel mundial, como se había ya señalado. De hecho, según las estadísticas que ofrece la propia plataforma, cada día se ven cientos de millones de horas de vídeos y se generan miles de millones de reproducciones (5).

Además, cada vídeo cuenta con su propio contador de visitas que permite dar a conocer abiertamente el número de visionados y/o la popularidad del mismo. Si bien, el público expectante puede encontrar particularmente alguna característica que hace que algunos contenidos sean ampliamente difundidos, de forma espontánea, a través de otras redes sociales convirtiéndose así en virales. Pero, un trabajo (12) que evaluó las características de los vídeos relacionados con los cuidados al final de la vida en YouTube no identificó ningún video viral sobre esta temática. En general, solo el 1\% de los videos en YouTube alcanzan la categoría de «viral» (más de 5.000 .000 consultas en un mes de existencia en la plataforma) (13).

En cuanto a la participación de los usuarios en los vídeos sobre «cuidados paliativos en el domicilio", los datos obtenidos en este estudio indican que la participación activa de los mismos, basados en las indicaciones favorables/desfavorables, los comentarios realizados o las suscripciones, indican una baja interacción (menos de 1 por cada 1.000 reproducciones). Si bien, resaltar que en el caso de las indicaciones favorables esta cifra es ligeramente superior (6).

Hay que tener en cuenta que, para participar en las opciones de interacción que ofrece YouTube, el usuario necesita registrarse (de manera gratuita) en la plataforma y que mediante el modelo de votación 'me gusta' (dedo pulgar hacia arriba) y 'no me gusta' (dedo pulgar hacia abajo), los internautas que consumen material multimedia en Internet adoptan una actitud pasiva heredando así el comportamiento que tienen como espectadores ante los medios unidireccionales y tradicionales como la televisión (2). No estamos planteando nada que no se haya advertido previamente. Así, investigadores como Owen (14) aseguran que la pasividad es algo atractivo para el espectador en el proceso de comunicación.

El hecho de que un internauta pueda elegir lo que quiere ver y cuándo lo quiere ver, no implica que hayan alcanzado mayores cuotas de interacción sino que ese tipo de interacción se da por hecho que va implícita con el carácter del internauta (2).

El origen y autoría de los vídeos sobre cuidados paliativos en el domicilio estudiados, denotan un claro predominio de los contenidos subidos por profesionales socio-sanitarios y personas privadas. Destaca, a su vez, la presencia de numerosos vídeos con autoría de los medios de comunicación clásicos o de masas que han trasladado su poder e influencia a Internet, manteniendo una destacable cuota de mercado en el consumo de vídeo en la Red. Por el contrario, llama la atención la baja participación en la edición de contenidos del mundo académico o el casi nulo de las sociedades médicas. De todos modos, la participación de los profesionales en la edición de contenidos en la Web 2.0, que no en su consulta, ya ha sido estudiado en trabajos previos (15).

Según Pisani, profesor de la Universidad de Berkeley (California), «los medios tradicionales tienen que enfrentarse al desafío que supone una audiencia cada vez más preparada y más experta en el consumo de información» (16). Claro ejemplo es el exitoso formato de YouTube, que hace que el usuario de la Web 2.0 comience a descubrir una nueva forma de disfrutar de los productos 
audiovisuales, descontextualizándolos del resto de contenidos con los que se emiten en los medios tradicionales, es decir, el usuario tiene la capacidad para decidir a qué informaciones acceder o no, qué valoración merecen y si conviene, o no, comentar algún aspecto de la noticia. Todo esto permite a los internautas jerarquizar el interés informativo de los distintos contenidos que se ofrecen (17).

En cuanto a la duración de los vídeos de carácter formativo cabe señalar que está en línea con los estudios de Sexton (18), que señalaban que no conviene que los vídeos destinados a la formación tengan una duración superior a los 5 minutos; conociéndose como "píldora educativa".

El estudio demuestra que la mayoría de vídeos de Internet acerca de los cuidados paliativos en el domicilio ha encontrado, en general, resultados similares a los estudios realizados, en el pasado, sobre el papel de las redes sociales, y en particular de YouTube, como fuente de información en salud pública, como el brote epidémico del virus Ébola (19), la gripe H1N1 (20) y la inmunización (21) que se caracterizaron, en general, por una información de pobre calidad o irrelevante, e incluso en ocasiones engañosa, solo algunos vídeos contienen información de alta calidad.

Dado que el contenido de YouTube puede ser subido a la plataforma por cualquier usuario de la misma, sería de gran ayuda, para los usuarios menos instruidos, el contar con indicaciones que garantizasen la calidad de la información. Pero, la baja interacción de usuario del mundo científico y académico no permite asegurar, hoy por hoy, esta iniciativa.

La muerte y el morir han sido considerados en un pasado no muy lejano como un tema tabú, pero la voluntad y la necesidad de hablar abiertamente sobre cómo afrontarlo, incluso en la Red, parecen estar en aumento. Las redes sociales, como YouTube, hasta cierto punto, han traído el dolor y la atención al final de la vida de la esfera del ámbito privado a la vida cotidiana del omnipresente mundo digital. Así, la forma en que los pacientes con enfermedades terminales y sus familias se acercan a los últimos años, meses y semanas de sus vidas y cómo estos podrían esperar que los profesionales de CP trabajen e interactúen con ellos durante estos difíciles momentos puede tener un impacto significativo en estas personas (22).

El único objetivo de un buen CP debe ser mantener la mejor calidad de vida posible para el paciente y su familia justamente en el espacio donde ellos eligen estar, en su domicilio. Así pues, dar a conocer los CP, "promocionarlos" en la población general a través de los diferentes medios de comunicación es una cuestión necesaria (23).

Estos resultados con respecto a la temática a estudio, ponen de manifiesto, en la misma línea de trabajos anteriores $(12,24)$, que son necesarios futuros estudios para entender si esta herramienta, así como qué características de los vídeos pueden 'enganchar' a pacientes, y al público en general, con este problema de salud pública (25), a la vez que suponen un reto para los profesionales de este campo.

El uso de la Web social supone un desafío pero también presenta grandes oportunidades. Sería necesaria una cuidadosa discusión acerca de sus límites y limitaciones pero, también, una mente abierta hacia el cambio social que representan y cómo encajarlo en los CP (22).

Por tanto, la difusión de información online sobre cuidados paliativos en el domicilio en YouTube presenta ventajas indiscutibles, como su bajo coste, su enorme potencial de hacer llegar información rápidamente a un amplio sector de la población o el hecho de facilitar la interacción con los usuarios pero, también, numerosos inconvenientes ya que en muchos casos no puede identificarse al autor del vídeo, no se citan fuentes, se muestran opiniones personales como si fueran hechos científicos o algunos aspectos quedan sin respuesta, por lo que puede ser difícil para el usuario no experto valorar la calidad de sus contenidos $(26,27)$.

Por otra parte, la presencia de mensajes interpuestos en los vídeos estuvo en consonancia con un estudio de la información en varios mass media españoles en relación con este tipo de atención (28). 
Finalmente, en cuanto a YouTube como plataforma publicitaria, desde mayo de 2007 empezaron a aparecer vídeos publicitarios 'saltables o no' y otros en estilo pop-up. El anuncio puede aparecer al principio (pre-roll), en algún punto intermedio (mid-roll) o al final del vídeo (post-roll). El tipo de anuncio mostrado al usuario es completamente aleatorio y sin relación con el contenido del que vídeo que buscaba ver o las palabras clave usadas en la búsqueda. El usuario/generador de contenidos estándar de YouTube puede desactivar que sus propios vídeos tengan anuncios de terceros $(29,30)$.

Algunas limitaciones podrían derivarse de la actualidad/obsolescencia: los propios datos obtenidos en este trabajo indican que la rápida obsolescencia de los resultados pueden dar una imagen algo distorsionada de la realidad actual y obligaría a realizar estudios de seguimiento de la calidad de la información, en este caso sobre cuidados paliativos en el domicilio.

Por otro lado, al seleccionar los vídeos al azar, puede ser que estos no se correspondan con los más utilizados. En todo caso, lo que este trabajo ha querido representar es una búsqueda de información libre. Lo que coloquialmente se entiende por 'navegar'. Es decir, la pesquisa no dirigida ni realizada por un experto en la materia. Al trabajar con asignación muestral se ha intentado evitar la subjetividad del usuario relacionada con el posicionamiento de los resultados: la consulta tan solo de los primeros vídeos. De todos modos, para evitar en lo posible este sesgo, se han seleccionado todos los vídeos de la primera pantalla.

El contenido es clave, pero si el mensaje no es claro y atractivo, difícilmente llegará a la población objetivo. Hay que insistir en que todo lo que hay en la Red no es bueno y en que queda mucho por hacer a la hora de educar o de dar respuestas sobre temas de salud a los usuarios.

Por todo lo expuesto, se puede concluir: Los indicadores del material multimedia son propios de la nueva comunicación online y relacionados con los nuevos estudios sobre Altmetría (Webmetría): gran producción y divulgación (consulta), rápida obsolescencia y una escasa participación de usuario. La mayoría de los videos analizados pueden considerarse adecuados con el tema a estudio.

\section{BIBLIOGRAFÍA}

1. Mekaru SR, Brownstein JS. One Health in social networks and social media. Rev Sci Tech Int Off Epizoot. 2014;33(2):629-37. PMID:25707189

2. Gallardo-Camacho J, Jorge-Alonso A. La baja interacción del espectador de vídeos en Internet caso Youtube España. Revista Latina de Comunicación Social. 2010;65:421-35. DOI: 10.4185/ RLCS-65-2010-910-421-435

3. Alexa, The Web Information Company [sede Web]. EE.UU.: Amazon.com Company. [consultado: 09 de mayo de 2017]. Top Sites (The top 500 sites on the Web) [aprox. 6 pantallas]. Disponible en: http://www.alexa.com/topsites

4. Gallardo-Camacho J. Análisis del fenómeno Youtube en España: Relación con los espectadores y con los generadores de contenidos tradicionales. Revista Luciérnaga. 2013;57-68.

5. YouTube [sede Web]. Apartado de prensa: Estadísticas-YouTube. [consultado: 20 de junio de 2017]. Disponible en: https://www.youtube.com/yt/press/es/statistics.html

6. Oller-Arlandis VE. Calidad y pertinencia de la información existente en la Web 2.0 sobre medio ambiente [Tesis doctoral] Sant Joan d'Alacant, Alicante, España: Departamento de Salud Pública, Historia de la Ciencia y Ginecología. Universidad Miguel Hernández de Elche; 2016. 
7. Konstantinidis S, Fernandez-Luque L, Bamidis P, Karlsen R. The role of taxonomies in social media and the semantic web for health education. A study of SNOMED CT terms in YouTube health video tags. Methods Inf Med. 2013;52(2):168-79. DOI: 10.3414 / ME12-02-0005; PMID: 23450378

8. Curbelo-Castro MC. Información sanitaria en la Web 2.0: YouTube como fuente de divulgación del conocimiento sobre lactancia materna [Tesis doctoral] Alicante, España: Departamento de Enfermería comunitaria, Medicina Preventiva, Salud Pública e Historia de la ciencia, Universidad de Alicante; 2015.

9. Guardiola-Wanden-Bergue R, Sanz-Valero J, Wanden-Bergue C. Eating Disorders Blogs: Testing the Quality of Information on the Internet. Eat Disord. 2010;148-52. DOI: 10.1080/10640260903585565; PMID: 20390618

10. Guardiola-Wanden-Bergue R, Gil-Pérez JD, Sanz-Valero J, Wanden-Bergue C. Evaluating the quality of websites relating to diet and eating disorders. Health Information and Libraries Journal. 2011;294-301. DOI: 10.1111/j.1471-1842.2011.00961.x; PMID: 22051128

11. Price DJ de S, Price DJ de S. Little science, big science-- and beyond. New York, USA: Columbia University Press; 1986. p 301

12. Mitchell IA, Schuster ALR, Lynch T, Smith KC, Bridges JFP, Aslakson RA. Why don't end-of-life conversations go viral? A review of videos on YouTube. BMJ Support Palliat Care. 2017;7(2):197204. DOI: 10.1136/bmjspcare-2014-000805; PMID: 26182948

13. West T. Going Viral: Factors That Lead Videos to Become Internet Phenomena. The Elon Journal of Undergraduate Research in Communications. 2001;2(1):76-84.

14. Owen BM. The Internet challenge to television. Cambridge, Massachussets, USA.: Harvard Univ. Press; 2000.

15. López Marcos P, Sanz-Valero J. Presencia y adecuación de los principios activos farmacológicos en la edición española de la Wikipedia. Aten Primaria. 2013;45(2):101-6. DOI: 10.106/j. aprim.2012.09.012; PMID: 23159792

16. Pisani F. El estado de los blogs periodísticos en la web 2.0. En: II Congreso Internacional de Blogs y periodismo en la Red Madrid, España Universidad Complutense de Madrid; Facultad de Ciencias de la Información. 25 y 26 de abril 2007 [citado: 20 de junio de 2017]. Disponible en: https://goo.gl/v3BLeC

17. Polo Serrano D. La comunicación a través del vídeo streaming: Análisis del caso YouTube [Tesis doctoral]. Granada, España: Departamento de Biblioteconomía y Documentación. Universidad de Granada; 2010.

18. Sexton R. Using short movie and television clips in the economics principles class. J Econ Educ. 2006;37(4):406-17.

19. Pathak R, Poudel DR, Karmacharva P, Pathak A, Aryal MR, Mahmood M, et al. Youtube as a source of information on Ebola virus disease. N Am J Med Sci. 2015;7(7):306-9. DOI: 10.4103 / 1.947-2714,161244; PMID: 26258077

20. Pandey A, Patni N, Singh M, Sood A, Singh G. YouTube as a source of information on the H1N1 influenza pandemic. Am J Prev Med. 2010;38(3):e1-3. DOI: 10.1016/j.amepre.2009.11.007; PMID: 20171526

21. Keelan J, Pavri-Garcia V, Tomlinson G, Wilson K. YouTube as a source of information on immunization: a content analysis. JAMA. 2007;298(21):2482-4. DOI: 10.1001/jama.298.21.2482; PMID: 18056901 
22. Taubert M, Watts G, Boland J, Radbruch L. Palliative social media. BMJ Support Palliat Care. 2014;4(1):13-8. DOI:10.1136/bmjspcare-2013-000584; PMID: 24644766

23. Fornells H. Cuidados Paliativos en el domicilio. Acta Bioethica. 2000;6(1):65-75.

24. Wittenberg-Lyles E, Parker Oliver D, Demiris G, Swarz J, Rendo M. YouTube as a Tool for Pain Management With Informal Caregivers of Cancer Patients: A Systematic Review. J Pain Symptom Manage. 2014;48(6):1200-10. DOI: 10.1016/j.jpainsymman.2014.02.015; PMID: 24793505

25. De Lima L, Pastrana T. Opportunities for Palliative Care in Public Health. Annu Rev Public Health. 2016;37(1):357-74. DOI:10.1146/annurev-publhealth-032315-021448; PMID: 26989831

26. Gabarrón E, Fernández-Luque L. eSalud y vídeos online para la promoción de la salud. Gac Sanit. 2012;26(3):197-200. DOI: 10.1016/j.gaceta.2012.03.004

27. Jamwal NR, Kumar SP. Maintaining the Social Flow of Evidence-Informed Palliative Care: Use and Misuse of YouTube. Indian J Palliat Care. 2016;22(1):104-7. DOI: 10.4103/ 0.9731.075,173945; PMID: 26962290

28. Carrasco JM, Centeno C. What message do Spanish media convey about palliative care? European Journal of Palliatice Care. 2016;23(1):32-4.

29. Wikipedia, la enciclopedia libre [Sede Web]. YouTube [aprox. 12 pantallas] [consultado: 20 de junio de 2017]. Disponible en: https://es.wikipedia.org/wiki/YouTube

30. YouTube [sede Web]. Cómo funcionan los anuncios de vídeo. [consultado: 20 de junio de 2017]. Disponible en: https://goo.gl/1EQMjb 